\title{
ESTIMATIVAS DAS FALHAS NO ESTANDE E PRODUTIVIDADE DO AMENDOIM UTILIZANDO INFRAVERMELHO PRÓXIMO
}

Joissy M. de A. Andrade ${ }^{1}$; Denis R. Pretto ${ }^{2}$; Denizart Bolonhezi ${ }^{3}$; Jose Roberto Scarpellini ${ }^{4}$ Eglairto V. Carvalho ${ }^{5}$; Bruna Cardoso Vieira ${ }^{6}$

${ }^{1}$ Mestranda em Agronomia (Ciência do Solo), FCAV/UNESP, Jaboticabal, SP e Analista de Suporte Tecnico,

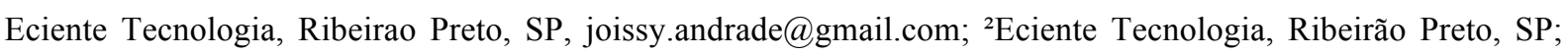
${ }^{3}$ Pesquisador Cientíco, IAC/Centro de Cana, Bolsista do CNPQ, Ribeirão Preto; ${ }^{4}$ Agência Paulista de Tecnologia dos Agronegócios - APTA/SAA; ${ }^{5}$ Eciente Tecnologia, Ribeirão Preto, SP; ${ }^{6}$ Bolsista Iniciação PIBIT/CNPQ Agência Paulista de Tecnologia dos Agronegócios.

RESUMO: O presente estudo tem o objetivo estudar o uso de imagens NIR para estimativa de produção de amendoim em três diferentes processos de Plantio (plantio convencional, plantio direto e plantio Rip Strip) relacionando informações de produtividade com parâmetros extraídos de Ortofoto gerada por um Veículo Aéreo Não Tripulado (VANT). As imagens em escala de erro de 0,2 cm foram analisadas em extratos compostos de duas linhas centrais lançados em todas as repetições dos três diferentes tratamentos. Para cada linha, com 100 metros lineares foram medidas e quantificadas a existência de falhas; posteriormente determinou-se o efetivo plantio verificado em cada tratamento e correlacionou-se com a produção obtida nos tratamentos. As linhas de plantio e as falhas identificadas, foram analisadas com o Quantum Gis. Os resultados da correlação tenderam a uma correlação positiva entre os dados de falhas e massa foliar e produtividade, sendo um indicador positivo de uso desta ferramenta para medições de produtividade através de NIR.

Palavras-chave: Amendoim, Processamento Imagem, SIG, VANT.

\section{INTRODUÇÃO}

No Brasil, as primeiras pesquisas sobre manejo conservacionista do solo para amendoim sobre cana crua foram realizadas pelo IAC em Ribeirão Preto. Estas pesquisas concluíram que: a nodulação é duas vezes maior, a umidade do solo na zona de crescimento das vagens é $18 \%$ maior, que o controle de plantas daninhas é favorecido e a produtividade de vagens não é reduzida no plantio direto de amendoim (BOLONHEZI et al., 2007). Tais resultados são importantes na atualidade, considerando que, a partir de 2017, todos os canaviais paulistas devem ser colhidos sem queima prévia, demandando assim, mais gastos com operações de preparo do solo. O custo pode aumentar em até $30 \%$. Em virtude de mais de $80 \%$ da produção de amendoim estar em reforma de canaviais, os produtores forçosamente deverão adotar sistemas de manejo conservacionistas. Mas existem diversas dúvidas que ainda necessitam de esclarecimento, sobretudo nas operações de colheita, devido as peculiaridades na morfologia e na fisiologia dessa oleaginosa. 
Um equipamento vindo dos EUA, foi introduzido, sendo que este efetua preparo somente na faixa de semeadura, conhecido como "Rip Strip". Este equipamento realiza preparo em faixas, entre 20 e $46 \mathrm{~cm}$ de largura através de quatro discos corrugados posicionados na vertical e entre 25 e $45 \mathrm{~cm}$ de profundidade, através de uma haste subsoladora. SIRI-PRIETO et al. (2009) estudaram o uso deste implemento em comparação com a semeadura direta, em integração com pecuária. Concluíram que o maior lucro foi obtido com o preparo em faixa (US\$ 462), em comparação à semeadura direta sobre pastagem (US\$ 41).

O interesse em Veículos Aéreos Não Tripulados (VANT) tem crescido ao redor do mundo. Avanços recentes na tecnologia computacional, desenvolvimento de software, materiais mais leves, sistemas globais de navegação, avançados links de dados, sofisticados sensores e a miniaturização são os motivos do aumento de desenvolvimentos de VANTs. Os Estados Unidos aparecem como um dos líderes em termos de tamanhos, tipos e sofisticações dos sistemas, que são voltados principalmente para o mercado militar. Dentre os outros países que têm procurado desenvolvimento e aplicações de VANTs, o Japão se destaca com mais de 2000 VANTs aplicados em pulverização e outras aplicações na agricultura (SIMPSON, 2003; DE GARMO, 2004).

Técnicas computacionais, sensoriamento remoto e sistemas de posicionamento global são utilizados em conjunto para o desenvolvimento de ferramentas que auxiliam na gestão agrícola, podendo assim, obter através desses sistemas dados que auxiliam fortemente na agricultura de precisão. Existem diversas técnicas de processamento digital de imagens provenientes de sensoriamento remoto, dentre elas está a classificação de imagens, que é uma técnica utilizada para extração de informações a respeito de determinados alvos utilizando-se do agrupamento de pixels em classes ou temas específicos (ERDAS, 1999). Na agricultura, as utilizações de sensores existem para que sejam capturadas as ondas presentes em cada banda da imagem, em que, cada banda: verde, vermelho e infravermelho próximo (NIR), permitem verificar o estado nutricional da cultura, através da análise do comportamento espectral.

Existem vários desafios e perspectivas e para a agricultura destacando-se o monitoramento de recursos naturais, meio ambiente, atmosfera, imageamento hiperespectral, observações de rios e lagos, bem como o imageamento de práticas agrícolas e uso do solo (JORGE et al., 1999; JORGE, 2001; JORGE, 2003; EISENBEISS, 2004). A aplicação de processamentos computacionais na área agrícola permite o desenvolvimento de softwares, onde cada qual possa suprir as necessidades de informação para o agricultor, resultando em grandes benefícios ao setor agropecuário.

\section{MATERIAL E MÉTODOS}

A pesquisa teve por objetivo avaliar o uso de imagens NIR na estimativa de produção de amendoim (Arachis hypogaea L.) adquiridas por VANTS nos processos de plantio convencional, plantio direto e plantio Rip Strip. 
Em canavial localizado em Pitangueiras/SP, com solo classificado como Latossolo Vermelho, típico, eutrófico e textura argilosa, foram instalados três tratamentos de manejo; preparo convencional, preparo em faixa (Rip Strip) e semeadura direta. Foram utilizadas seis operações de preparo de solo (1 $\mathrm{x}$ gradagem aradora, $1 \mathrm{x}$ aração com aiveca, 2 x gradagens intermediárias $\mathrm{e} 1 \mathrm{x}$ enxada rotativa). Devido não possuir semeadora com os requisitos mínimos para semear sobre palhada de cana crua, foi convidada a empresa JUMIL no sentido de emprestar a semeadora 7090 Guerra GII. As operações de preparo iniciaram-se no dia 25/11/2015 e finalizaram com o tratamento "Rip Strip" que foi efetuado em 27/11/2015. A semeadura nos tratamentos convencional e "Rip Strip" foi realizada em 28/11/2015 e foi utilizada a mesma semeadora utilizada pelo produtor. No sistema "Rip Strip", houve necessidade de utilizar trator equipado com piloto automático, permitindo a coincidência da linha de semeadura com a faixa preparada. Devido à chuva, a semeadura do tratamento semeadura direta foi realizada no dia 03/12/2015. Foram utilizadas sementes do cultivar IAC-OL3, com densidade de semeadura de 20 sementes por metro e espaçamento de $0,90 \mathrm{~m}$. As sementes (categoria $\mathrm{C} 1$, peneira 25) dessa cultivar de característica alto oleica e ciclo de 125 dias foi fornecida pela COPLANA. As glebas destinadas para a instalação totalizaram 9,08 hectares. Os tratamentos foram arranjados em delineamento blocos ao acaso, com sete repetições.

Foram realizadas coletas do número de plantas por metro linear em unidades amostrais de 0,5m bem como, a medição de altura de plantas e massa vegetativa. Aos 20 dias do plantio foi realizado voo com VANT, de fabricação francesa, da marca Delair-Tech, modelo DT18, com câmera multiespectral acoplada, do próprio fabricante, permitindo capturas em cinco bandas: R (red), G (green), B (blue), NIR (near infrared reflectance), Red-Edge obtendo imagens georeferenciadas com pixel de 5,4cm de resolução. As fotos foram ortorretificadas, e processadas no software Pix 4d para obtenção da banda NIR, que é a energia radiante do infravermelho (IV) e é empregada para caracterizar substâncias orgânicas, cujo espectrômetro NIR se baseia na aplicação da matemática à química analítica (quimiometria).

Foi utilizado o Quantum Gis para medir as linhas de amostragens. Definiu-se a delimitação de duas linhas centrais com comprimento de 100 metros para o cálculo do efetivo plantio. O cálculo do efetivo plantio consistiu na medição do comprimento de linhas de plantio e contabilização de falhas na linha, aplicando o cálculo de comprimento. 


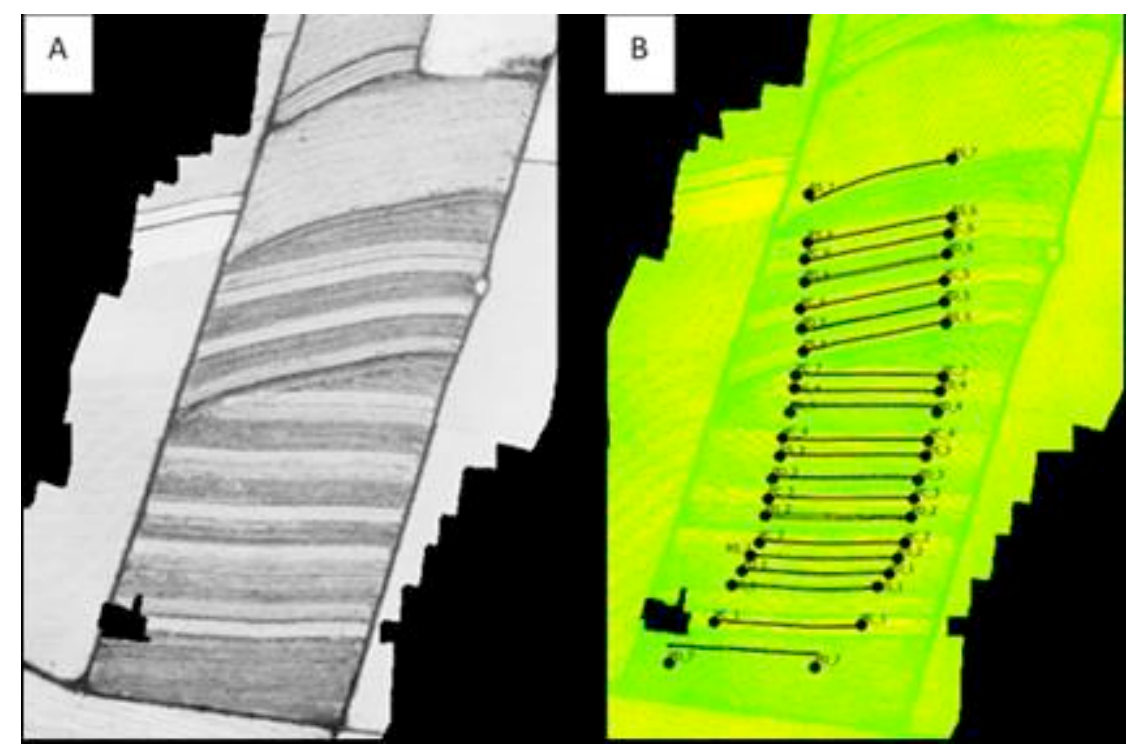

Figura 1. A) Ortofoto Processada banda NIR e B) Ortofoto com demarcações de pontos e linhas correspondentes as áreas amostradas para cada uma das repetições.

\section{RESULTADOS E DISCUSSÃO}

A Tabela 1 mostra que os tratamentos plantio convencional e Rip Strip apresentaram valores de efetivo plantio semelhante, havendo um diferencial na massa vegetativa com maior índice para o plantio convencional.

Tabela 1. Dados amostrados através de informações obtidas em imagem.

\begin{tabular}{|c|c|c|c|c|c|c|}
\hline \multicolumn{7}{|c|}{ TIPOS DE TRATAMENTOS } \\
\hline \multirow[b]{2}{*}{$\begin{array}{c}\text { REPETIÇÕ } \\
\text { ES }\end{array}$} & \multicolumn{2}{|c|}{$\begin{array}{c}\text { PLANTIO } \\
\text { CONVENCIONAL }\end{array}$} & \multicolumn{2}{|c|}{ PLANTIO DIRETO } & \multicolumn{2}{|c|}{ RIP STRIP } \\
\hline & $\begin{array}{c}\text { COMPRIMENTO } \\
\text { (M) }\end{array}$ & $\begin{array}{c}\text { Falhas } \\
\text { (M) }\end{array}$ & $\begin{array}{c}\text { COMPRIMENTO } \\
\text { (M) }\end{array}$ & $\begin{array}{l}\text { Falhas } \\
\text { (M) }\end{array}$ & $\begin{array}{l}\text { COMPRIMENTO } \\
\text { (M) }\end{array}$ & $\begin{array}{l}\text { Falhas } \\
\text { (M) }\end{array}$ \\
\hline 1 & 204,60 & 1,01 & 205,99 & 29,00 & 205,36 & 14,53 \\
\hline 2 & 203,49 & 0,14 & 202,24 & 70,45 & 204,75 & 4,88 \\
\hline 3 & 202,87 & & 204,35 & 33,83 & 204,48 & 2,42 \\
\hline 4 & 203,73 & & 204,93 & 57,12 & 203,34 & 1,41 \\
\hline 5 & 204,17 & 0,60 & 204,38 & 39,98 & 203,65 & 5,60 \\
\hline 6 & 204,30 & 0,84 & 204,12 & 8,48 & 203,45 & 4,67 \\
\hline 7 & 203,37 & & 211,28 & 67,07 & 207,18 & 2,63 \\
\hline $\begin{array}{l}\text { TOTAL } \\
\text { (M) }\end{array}$ & 1426,52 & 2,59 & 1437,27 & 305,94 & 1432,22 & 36,16 \\
\hline
\end{tabular}




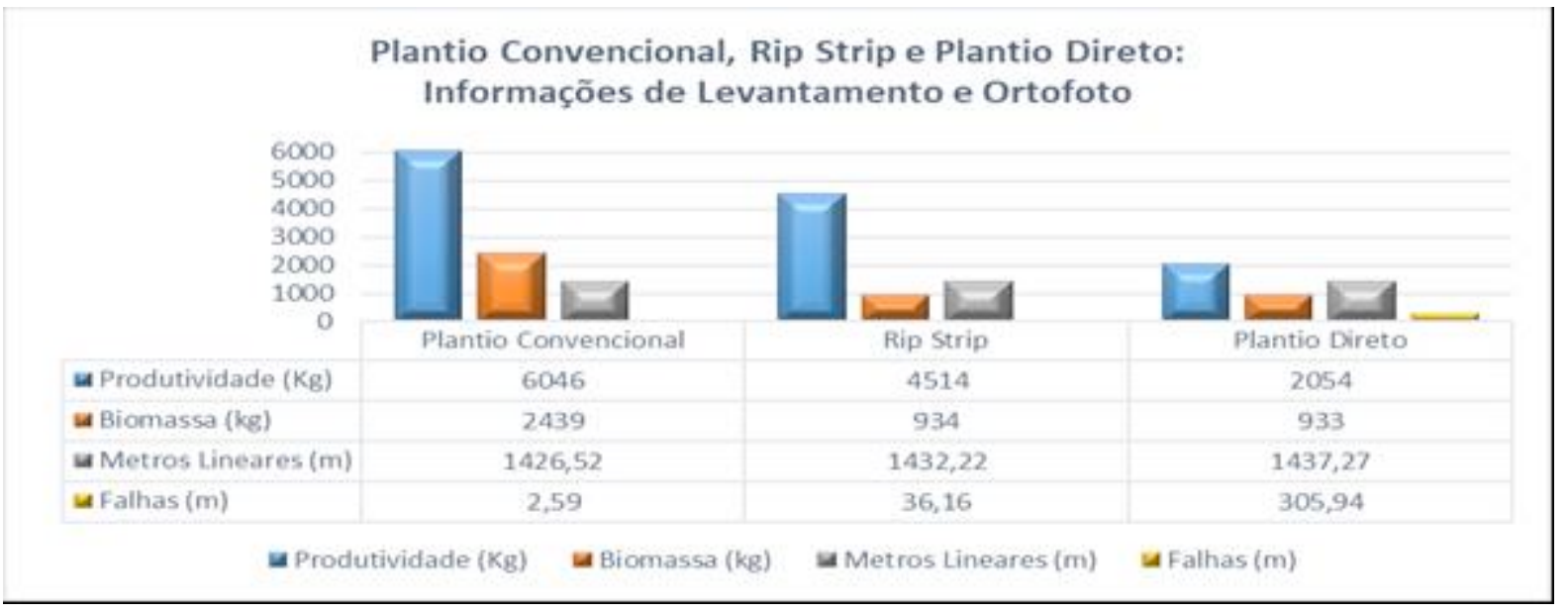

Gráfico 1: Informações de levantamento e ortofoto, biomassa da parte aérea aos 45 dias e produtividade na colheita, nos três sistemas de manejo.

No gráfico 1 pode-se observar os indicadores de produtividade dos diferentes tratamentos e associando-se aos resultados de imagem NIR obtidos pode-se inferir que este espectro pode ser aprimorado para uso na estimativa de produtividade da cultura do amendoim considerando efetivo plantio e massa vegetativa.

Os dados da tabela abaixo (Tabela 2) permite visualizar a relação encontrada entre as amostragens realizadas por tratamento pelos metros de falhas encontrados em cada um dos plantios utilizados.

Tabela 2. Relação entre amostragem realizada por tratamento por falhas encontradas em cada tratamento (metros lineares).

\begin{tabular}{|cccc|}
\hline & $\begin{array}{c}\text { PLANTIO } \\
\text { CONVENCIONAL }\end{array}$ & $\begin{array}{c}\text { PLANTIO } \\
\text { DIRETO }\end{array}$ & RIP STRIP \\
\hline $\begin{array}{c}\text { Relação entre: Amostragem por } \\
\text { tratamento (metros lineares) } \\
\text { Falhas (metros lineares) }\end{array}$ & $0,18 \%$ & $21,29 \%$ & $2,52 \%$ \\
\hline
\end{tabular}

\section{CONCLUSÃO}

As imagens geradas através do VANT identificaram com grande precisão as falhas ocorridas no campo aos 45 dias após a semeadura, as quais permitiram discriminar os tratamentos de manejo com acurácia. As falhas quantificadas no plantio direto foram 20 vezes maiores que no sistema de preparo convencional.

O uso de imagens NIR pode ser uma excelente ferramenta para estimativa de produção da cultura do amendoim, havendo a necessidade de estudos mais detalhados contemplando diferentes condições de solo, sistemas de cultivo e variedades. 


\section{REFERENCIAS BIBLIOGRÁFICAS}

BOLONHEZI, D.; MUTTON, M.A.; MARTINS, A.L.M. Sistemas conservacionistas de manejo do solo para amendoim cultivado em sucessão à cana crua. Pesquisa Agropecuária Brasileira, Brasília, v.42, n.7, p. 939-947, 2007.

DE GARMO, M. T. Issues Concerning Integration of Unmanned Aerial Vehicles in Civil Airspace. Center for Advanced Aviation System Development - Mitre, McLean, Virginia. 2004.

EISENBEISS, H. A mini unmanned aerial vehicle (UAV): system overview and image acquisition. International Workshop on "Processing and visualization using high resolution imagery". Pitsanulok, Thailand. Nov. 2004.

ERDAS. Field guide. 5. ed. Atlanta - EUA: ERDAS, p. 672, 1999.

JORGE, L. A. C.; Souza, N. P.; Trindade Junior, O.; Tozzi, C. L.; Torre Neto, A. Studies of Soil Conservation for Precision Farming by Digital Color Image ANALYSIS. In: 1999 ASAE Annual International Meeting, 1999, Toronto, Ontário Canada. Anais do ASAE Annual International Meeting, 1999.

JORGE, L.A.C. Determinação da cobertura de solo em fotografias aéreas do Projeto Arara. Dissertação (Mestrado em Ciências da Computação) Universidade de São Paulo, São Carlos, 97f, 2001.

JORGE, L.A.C. Metodologia para utilização de aeromodelos em monitoramento aéreo: análise de imagens. EMBRAPA Instrumentação Agropecuária, São Carlos, SP, Brasil. Circular Técnica 18 de Novembro de 2003.

SIMPSON, A. D. Development of an unmanned aerial vehicle for low-cost remote sensing and aerial photography. Master of Science Thesis. University of Kentucky, Lexington, Kentucky. 2003.

SIRI-PRIETO, G.; Revees, D. W.; Raper, R. L.Tillage Requirements for Integrating WinterAnnual Grazing in Peanut Production: Plant Water Status and Productivity. Agronomy Journal, v.101, n.6, p.1400-1408, 2009. 